\title{
Encephalopathy associated with autoimmune thyroid disease: a rare but frequently missed neuropsychiatric syndrome
}

\author{
Gaël Vermeersch ${ }^{1}$ id $\cdot$ Jen Maes $^{2}$ \\ Received: 7 March 2021 / Accepted: 13 April 2021 / Published online: 18 April 2021 \\ (c) Belgian Neurological Society 2021
}

Keywords Hashimoto encephalopathy $\cdot$ Autoimmune encephalopathies $\cdot$ Autoimmune thyroid disease $\cdot$ SREAT

\section{Dear Editor,}

We report the case of a patient with "encephalopathy associated with autoimmune thyroid disease" (EAATD) diagnosed and treated in our hospital. As EAATD is poorly understood and has no pathognomonic features, the diagnosis remains challenging.

\section{Case}

In July 2020, a 75-year-old Caucasian woman consulted our neurology department due to motoric deterioration and progressive cognitive decline since several weeks. In 2019, the patient had been hospitalised elsewhere due to similar symptoms; no final diagnosis was formulated in the medical file. Brain imaging performed during this latter hospitalisation resulted normal. Aside from hypothyroidy, medical history included no prominent abnormalities. Her medical therapy existed out of duloxetine, bromazepam, pantoprazole, dosulepin and L-thyroxine (125 $\mu \mathrm{g} /$ day $)$. Increased levels of thyroid-stimulating hormone (6.3 mU/L, RR 0.27-4.20) and TPOAbs $(>600 \mu / \mathrm{mL}, \mathrm{RR}<60.0)$ were the most remarkable biochemical findings. Urine culture and broad screening for infections resulted normal. Brain imaging was not performed at this stage. The tentative diagnosis of EAATD was made and oral methylprednisolone (64 mg/day), with an increased dose of L-thyroxine (150 $\mu \mathrm{g} /$ day), was initiated. The clinical

Gaël Vermeersch

gael.vermeersch@student.kuleuven.be;

gael.vermeersch@telenet.be

1 Faculty of Medicine, KU Leuven, Herestraat 49, 3001 Leuven, Belgium

2 Department of Neurology, AZ Delta Campus Rembert Torhout, Sint-Rembertlaan 21, 8820 Torhout, Belgium status significantly improved within 2 weeks. The encephalopathy appeared to be corticoid dependent as tapering was followed by reduced cognitive and functional ability. After initiating mycophenolate mofetil $(2 \times 500 \mathrm{mg} /$ day $)$, a steroid dose of $8 \mathrm{mg} /$ day was obtained stepwise.

In November 2020, she was hospitalised with "coronavirus disease-2019". As mycophenolate had to be stopped, relapse of EAATD was observed. The patient initially responded well when we increased methylprednisolone to $32 \mathrm{mg} /$ day. However, there was no full recovery. Brain Magnetic Resonance Imaging (MRI) showed no abnormalities and analysis of the cerebrospinal fluid (CSF) showed no signs of infection. Screening for Alzheimer's Disease (AD) showed reduced concentration of $\beta$-amyloid protein $1-42$ and of $\beta$-amyloid $1-42 / 1-40$ ratio (respectively, $16 \mathrm{pg} / \mathrm{mL}$, $\mathrm{RR}>824$ and $0.073, \mathrm{RR}>0.12$ ). Total concentration of tau protein and phosphorylated-tau resulted normal.

The clinical status further improved when methylprednisolone was increased to $48 \mathrm{mg} /$ day and mycophenolate $2 \times 500 \mathrm{mg} /$ day was restarted. Six weeks after admission, the patient was able to leave the hospital, the Mini-Mental State Examination (MMSE) at the time of discharge scored 22/30 and increased to 24/30 during follow-up. Positron Emission Tomography (PET)-CT, performed to exclude $\mathrm{AD}$ as a confounding factor in the MMSE score, resulted normal. During follow-up, the patient mentioned persistent diarrhoea, consequently mycophenolate was switched to azathioprine (150 mg/day). However, as the MMSE decreased to $17 / 30$ and steroid tapering failed, we stopped azathioprine and initiated plasmapheresis in March 2021. Currently, the MMSE recovered and methylprednisolone could be successfully reduced to an alternate-day dosing regimen of $16 \mathrm{mg}$ and $8 \mathrm{mg}$. Further reduction is foreseen. 


\section{Discussion}

EAATD, also known as "steroid responsive encephalopathy associated with autoimmune thyroiditis" (SREAT) or "Hashimoto encephalopathy", has a prevalence of approximately 2:100.000 and tends to be four times as prevalent in females compared to males [1]. The mean age of presentation in adults is $45-55$ years. Generally, EAATD is divided into stroke-like and indolent phenotypes; mixed presentations are possible.

Symptoms vary from tremor, headaches and sleep abnormalities to hallucinations, transient aphasia, cognitive impairment, hemiplegia or even coma and status epilepticus. The pathophysiology remains unknown, proposed etiologies include cerebral vasculitis, toxicity of thyrotropinreleasing hormone or an autoimmune reaction caused by shared epitopes in the thyroid gland and the central nerve system (CNS). Whether anti-thyroid antibodies play a direct etiopathogenic role is unclear. No relationship between antibody titre and disease severity is observed $[1,2]$. EAATD is frequently associated with the presence of TPOAbs, however, other antibodies such as antithyroglobulin and antialpha-enolase antibodies have been reported as well. Thus, EAATD is not exclusively related to Hashimoto's thyroiditis. EAATD tends to occur more frequently in euthyroid or mild hypothyroid status, nevertheless thyroid status does not appear to influence disease severity. No disease-specific findings are observed on MRI/CT or encephalography (EEG). Widespread slowing is the most common finding on EEG, which adds little to the diagnosis [1-4].

In general, EAATD responds well to treatment. $50 \%$ of the patients show complete remission after initiation of corticosteroids (frequently intravenous methylprednisolone $500-1000 \mathrm{mg} /$ day for 3-5 days; combined with oral therapy (1-2 mg/kg/day)). Oral steroids can be administrated concomitantly with intravenous therapy or be initiated afterwards. $60 \%$ of the patients show patterns of relapsing-remitting EAATD [2, 4]. Some patients appear to be steroid-resistant. If steroid monotherapy fails, or dose reduction is desired, other immunomodulators such as azathioprine, methotrexate, cyclophosphamide, rituximab or mycophenolate may be initiated. The duration of therapy is empirical, maintenance therapy is usually withdrawn after a symptom-free period of 3 years $[1,2]$.

As our reported patient clinically responded to the administration of corticosteroids, the diagnosis of EAATD is very presumable. The concomitant diagnosis of AD is unlikely at this stage as the PET-CT resulted normal, and further follow-up is desired. Few other case reports previously mentioned a peculiar CSF biomarker profile in patients with steroid-responsive encephalopathy [5].

\section{Conclusion}

EAATD should be considered in any patient with (sub) acute encephalopathy of unknown origin or in patients with progressive cognitive decline. No diagnostic test is available; CT/MRI and lumbar puncture should be performed to exclude infection, stroke or malignancy. Most patients with EAATD respond well to steroid therapy, however, secondline immunomodulators may be necessary.

Acknowledgements We thank the family and the person whose details are described here for their permission to publish this report.

Funding None.

\section{Declarations}

Conflict of interest The authors declare no financial or other conflicts of interest.

Ethical approval This article does not contain any studies with human participants or animals performed by any of the authors.

Informed consent Informed consent was obtained.

\section{References}

1. Montagna G, Imperiali M, Agazzi P, D’Aurizio F, Tozzoli R, Feldt-Rasmussen U et al (2016) Hashimoto's encephalopathy: a rare proteiform disorder. Autoimmun Rev 15:466-476. https://doi. org/10.1016/j.autrev.2016.01.014

2. Laurent C, Capron J, Quillerou B, Thomas G, Alamowitch S, Fain O et al (2016) Steroid-responsive encephalopathy associated with autoimmune thyroiditis (SREAT): characteristics, treatment and outcome in 251 cases from the literature. Autoimmun Rev 15:1129-1133. https://doi.org/10.1016/j.autrev.2016.09.008

3. de Holanda NCP, de Lima DD, Cavalcanti TB, Lucena CS, Bandeira F (2011) Hashimoto's encephalopathy: systematic review of the literature and an additional case. J Neuropsychiatry Clin Neurosci 23:384-390. https://doi.org/10.1176/jnp.23.4. jnp384

4. Zhou JY, Xu B, Lopes J, Blamoun J, Li L (2017) Hashimoto encephalopathy: literature review. Acta Neurol Scand 135:285290. https://doi.org/10.1111/ane.12618

5. Mateen FJ, Josephs KA, Parisi JE, Drubach DA, Caselli RJ, Kantarci K et al (2012) Steroid-responsive encephalopathy subsequently associated with Alzheimer's disease pathology: a case series. Neurocase 18:1-12. https://doi.org/10.1080/13554794. 2010.547503

Publisher's Note Springer Nature remains neutral with regard to jurisdictional claims in published maps and institutional affiliations. 